\title{
Does apolipoprotein E determine outcome of infection by varicella zoster virus and by Epstein Barr virus?
}

\author{
Matthew A Wozniak ${ }^{1}$, Suzanne J Shipley ${ }^{1}$, Curtis B Dobson ${ }^{1}$, Simon P Parker ${ }^{2}$, \\ Fiona T Scott ${ }^{2}$, Mary Leedham-Green ${ }^{2}$, Judy Breuer ${ }^{2}$ and Ruth F Itzhaki ${ }^{*, 1}$ \\ ${ }^{1}$ Faculty of Life Sciences, The University of Manchester, Manchester, UK; ${ }^{2}$ Skin Virology, Centre for Infectious Disease,
Institute of Cell and Molecular Science, Queen Mary University of London, London, UK
}

Over $90 \%$ of the population are infected with varicella zoster virus (VZV) but only some develop shingles caused when the virus reactivates from latency, and only some shingles patients develop post-herpetic neuralgia (PHN), defined as pain continuing for more than about 4 months. Epstein Barr virus (EBV) similarly infects over $90 \%$ of the population; some of those infected during teenage or young adult years develop infectious mononucleosis (IM). The reason for these disparities between numbers infected and numbers affected by illness is unknown, but presumably reflects host factor(s). Our previous results showed that apolipoprotein E (APOE) genotype determines susceptibility to, or outcome of, infection in the case of several diseases of known infectious cause. Therefore, we investigated APOE genotypes of shingles, PHN, and IM patients. Our rationale for the previous studies and for investigating VZV was that these micro-organisms use for cell binding and entry the same sites in the cell surface as does the protein apoE, and that consequently, competition with apoE could affect the pathogen's extent of entry and hence extent of the damage caused. The APOE genotypes of shingles and PHN sufferers, and of IM sufferers were determined using restriction fragment length polymorphism. In females, 84 homozygosity confers a risk of shingles and also of IM, and the APOE- $\varepsilon 4$ allele is protective against PHN whereas APOE- 83 allele is a risk. Our results showing that a host genetic factor influences the development of shingles and PHN in females have clinical significance: they could lead to identification of those (female) patients at greater risk of PHN, thus enabling these people to be targeted for treatment with the most effective drugs.

European Journal of Human Genetics (2007) 15, 672-678. doi:10.1038/sj.ejhg.5201812; published online 14 March 2007

Keywords: apolipoprotein E; Epstein-Barr virus; infectious mononucleosis; post-herpetic neuralgia; shingles; varicella zoster virus

\section{Introduction}

The outcome of infection with most pathogens varies, from asymptomatic effects to severe disease. For example, infection with varicella zoster virus (VZV) occurs in $90 \%$ of

${ }^{*}$ Correspondence: Professor RF Itzhaki, Faculty of Life Sciences, The University of Manchester, Manchester, UK.

Tel: +440161306 3879; Fax: + 440161306 4433;

E-mail: ruth.itzhaki@manchester.ac.uk

Received 21 December 2006; revised 3 February 2007; accepted 7 February 2007; published online 14 March 2007 the population, usually in childhood, and presents as chicken pox in the majority. The virus then remains latent in the sensory ganglia but can reactivate, causing shingles in some people. Furthermore, about $20 \%$ of shingles sufferers develop post-herpetic neuralgia (PHN) - pain that persists for $\geq 120$ days after the rash.

Epstein-Barr virus (EBV) infects $\sim 90 \%$ of the adult population too. In developed countries, primary infection occurs usually during adolescence or young adulthood and in $50 \%$ or more of such people, it causes infectious mononucleosis (IM). In contrast, in undeveloped countries, 
infection occurs usually in infancy and is normally asymptomatic.

The reasons for the differences in outcome of infection are largely unknown, but genetic factors presumably play a major role. We have found that polymorphisms in the gene encoding the protein apolipoprotein $\mathrm{E}$ (APOE) determine the consequences of infection with several diverse pathogens. This gene has three major alleles, APOE- $\varepsilon 2, \varepsilon 3$ and $\varepsilon 4$, which correspond to three main protein isoforms, apoE2, 3, and 4. The protein is a multipurpose molecule with roles in lipid transport, tissue repair and the immune system. ${ }^{1}$ We have identified a new function for apoE - control of pathogens. First, the APOE$\varepsilon 4$ and the APOE- $\varepsilon 2$ alleles are, respectively, risk factors for herpes labialis and herpes simplex encephalitis, diseases caused by herpes simplex virus type 1 (HSV1). ${ }^{2-4}$ Second, the APOE- $\varepsilon 4$ allele is strongly protective against hepatitis $\mathrm{C}$ virus-induced liver damage, ${ }^{5}$ and the APOE- $\varepsilon 2 \varepsilon 2$ genotype is associated with earlier infection with the malaria parasite. ${ }^{6}$ Also, we have discovered that a derivative of apoE has anti-infective properties. ${ }^{7}$ These findings all stem from our initial discovery that APOE- $\varepsilon 4$ and HSV1 (in brain) together confer a strong risk of Alzheimer's disease. ${ }^{2,3}$

The common factor linking apoE and these pathogens is their usage of the same molecules to attach to and/or to enter cells, namely, heparan sulphate proteoglycans (HSPG) and members of the low-density lipoprotein receptor (LDLR) family, respectively. ApoE might control infection by competing with the pathogen for binding to these molecules. ${ }^{2}$ If the extent of binding is isoformspecific, APOE will determine extent of competition, and hence of entry of the pathogen into cells and its subsequent spread, damage and disease. Dependence of binding affinities of the apoE isoforms on cell type could result in a specific allele protecting in one disease but conferring a risk in another.

VZV too attaches to $\mathrm{HSPG}^{8}{ }^{8}$ before binding to its specific receptor, insulin-degrading enzyme; ${ }^{9}$ thus APOE genotype might govern the outcome of infection with this virus. EBV, in contrast, uses neither HSPG nor the LDLR family, suggesting that its effects will not depend on APOE. Here, we describe our investigation of APOE polymorphism in disorders caused by these viruses.

\section{Methods \\ Patients}

We investigated APOE genotypes of 104 caucasian shingles sufferers (43 males, 61 females; mean age 59 years, range 19-91 years). Seventy were identified as having developed PHN, defined in this case as any pain or abnormal symptoms persisting for 120 days or more. The remaining subjects had no symptoms at 6 weeks. Initial diagnosis of shingles was made by the clinician on presentation of symptoms. Diagnosis was confirmed first by direct immunofluorescence with a VZV monoclonal antibody followed by multiplex polymerase chain reaction (PCR) for VZV and HSV. All patients were followed for at least 6 months. Patients were telephoned at 6,12 and 26 weeks and asked if they continued to suffer pain or abnormal sensations in the area of the original rash. Those who remained symptomatic were visited at home by a research nurse and their pain assessed by visual analogue score. The study was approved by the City and East London Ethics Committee and the Local Research Ethics Committee of the Redbridge and Waltham Forest Health Authority.

In the case of IM, 39 caucasians (18 males, 21 females; mean age 31 years, range 19-61) were recruited by advertising; they comprised mainly students and staff of the University of Manchester. All were selected by the criterion that they had at one time suffered from IM, which had been diagnosed by their general practitioner (GP). Informed consent was obtained in writing from each subject, under ethical approval from the Manchester Local Research Ethics Committee.

The control population comprised 238 volunteers (101 males, 132 females; mean age 37 years, and five of unspecified sex) from the University and from two local hospitals, as described previously. ${ }^{10}$ Among the University volunteer subjects, cold sore sufferers had been specifically sought in a previous study, ${ }^{3}$ and thus the proportion of sufferers was high and therefore non-representative of the general population. To compensate, the proportion of subjects with cold sores in the hospital survey, which was representative of the general population, was used to calculate appropriate genotype and allele frequencies for the University volunteers. The frequencies for the combined University plus hospital groups were then calculated on a weighted basis.

\section{DNA preparation and APOE genotyping}

For the shingles cases, DNA was prepared from serum using the Nucleospin tissue preparation kit (Abgene, Epsom, UK) according to manufacturers' instructions. Briefly, serum was subjected to proteinase $\mathrm{K}$ digestion and ethanol precipitation followed by binding to a column, washing and then elution.

For the IM sufferers, DNA was prepared from blood using the Nucleon BACC1 kit (Tepnel, Manchester, UK) according to manufacturers' instructions. Briefly, the white blood cells were isolated, their DNA extracted via cell lysis and then cleaned using sodium perchlorate, chloroform and Nucleon ${ }^{\circledR}$ resin, before ethanol precipitation and resuspension in sterile distilled water.

APOE genotyping was preformed using the method of Wenham et al ${ }^{11}$ but using Hotstartaq ${ }^{\circledR}$ (Qiagen, Crawley, UK) and agarose gel electrophoresis. 


\section{Statistical methods}

APOE allele and genotype frequencies are reported with their group percentages. Allele frequencies were compared using the Fisher exact and $\chi^{2}$ tests as appropriate. The risk associated with the presence of a particular APOE allele was estimated for each disease using odds ratios (with their 95\% confidence limits). Statistical significance was set at the conventional $5 \%$ level for all analyses.

\section{Results}

We determined APOE genotypes and allele frequencies of PHN and shingles sufferers who did not develop PHN (Table 1). The main statistically significant difference between these groups is an increased APOE- $\varepsilon 3$ allele frequency in the PHN sufferers, suggesting that this allele is a risk for this disease. Consistently, APOE- 83 homozygotes are more numerous in this group. Another statistically significant difference is a lower APOE- $\varepsilon 2 \varepsilon 3$ frequency in the subjects with PHN, suggesting that this genotype is protective against the disease and that possession of one $\varepsilon 2$ allele is sufficient to counter the harmful effects of an APOE- 83 allele. Again consistently, the APOE- $\varepsilon 2$ allele frequency is higher in the non-PHN than in the PHN sufferers, particularly in females, although neither difference reaches significance. Such heterozygote effects are difficult to explain, but in any case, caution should be taken when drawing conclusions from this result, as the number of APOE- $\varepsilon 2 \varepsilon 3$ possessors is very small.

Further interesting findings are revealed after the PHN and non-PHN groups are segregated by gender. First, the $\mathrm{APOE}-\varepsilon 3$ allele and APOE- $\varepsilon 3 \varepsilon 3$ genotype frequencies in female PHN sufferers are statistically significantly higher than in female non-sufferers. Second, female PHN sufferers have a lower APOE- $\varepsilon 4$ frequency than non-sufferers. (APOE- $\varepsilon 4 \varepsilon 4$ is lower for female PHN sufferers and this yields a $P$-value of $<0.05$ but the difference is not significant as the $95 \% \mathrm{CI}$ includes unity.) Third, for males there is no significant effect of APOE genotype or allele frequency between PHN and non-PHN groups. Thus, these data suggest that APOE- $\varepsilon 3$ confers a risk of PHN in females, APOE- $\varepsilon 4$ offers protection from PHN in females, and the effects seen in the whole group are due to those in females alone.

Table 1 APOE genotype (a) and allele (b) frequencies of shingles sufferers with and without PHN

\begin{tabular}{|c|c|c|c|c|c|c|c|c|c|c|c|c|c|}
\hline \multirow[b]{2}{*}{ (a) } & \multicolumn{2}{|c|}{$\varepsilon 2 \varepsilon 2$} & \multicolumn{2}{|c|}{$\varepsilon 2 \varepsilon 3$} & \multicolumn{2}{|c|}{$\varepsilon 2 \varepsilon 4$} & \multicolumn{2}{|c|}{$\varepsilon 3 \varepsilon 3$} & \multicolumn{2}{|c|}{$\varepsilon 3 \varepsilon 4$} & \multicolumn{2}{|c|}{$\varepsilon 4 \varepsilon 4$} & \multirow[b]{2}{*}{ Total people } \\
\hline & $\mathrm{N}$ & $\%$ & $\mathrm{~N}$ & $\%$ & $\mathrm{~N}$ & $\%$ & $\mathrm{~N}$ & $\%$ & $N$ & $\%$ & $\mathrm{~N}$ & $\%$ & \\
\hline PHN & 1 & 1.4 & 3 & $4.3^{\mathrm{a}}$ & 1 & 1.4 & 49 & $70.0^{b}$ & 15 & 21.4 & 1 & 1.4 & 70 \\
\hline No PHN & 0 & 0.0 & 6 & 17.6 & 0 & 0.0 & 16 & 47.1 & 10 & 29.4 & 2 & 5.9 & 34 \\
\hline PHN (female only) & 0 & 0.0 & 1 & 2.4 & 0 & 0.0 & 33 & $80.5^{c}$ & 7 & 17.1 & 0 & $0.0^{\mathrm{d}}$ & 41 \\
\hline PHN (male only) & 1 & 3.4 & 2 & 6.9 & 1 & 3.4 & 16 & 55.2 & 8 & 27.6 & 1 & 3.4 & 29 \\
\hline No PHN (male only) & 0 & 0.0 & 3 & 21.4 & 0 & 0.0 & 8 & 57.1 & 3 & 21.4 & 0 & 0.0 & 14 \\
\hline (b) & $\mathrm{N}$ & $2 \%$ & $\mathrm{~N}$ & $\%$ & $\mathrm{~N}$ & $\%$ & Allele total & & & & & & \\
\hline No PHN (female only) & 3 & 7.5 & 26 & 65.0 & 11 & 27.5 & 40 & & & & & & \\
\hline PHN (male only) & 5 & 8.6 & 42 & 72.4 & 11 & 19.0 & 58 & & & & & & \\
\hline No PHN (male only) & 3 & 10.7 & 22 & 78.6 & 3 & 10.7 & 28 & & & & & & \\
\hline
\end{tabular}

Footnotes display statistics only for the PHN values that differ significantly from the no $\mathrm{PHN}$ values, that is $P \leq 0.05$.

${ }^{\mathrm{a}} \mathrm{P}=0.02$, OR: $0.21(95 \% \mathrm{Cl} 0.05-0.89)$.

${ }^{\mathrm{b}} \mathrm{P}=0.02$, OR: $2.63(95 \% \mathrm{Cl} 1.13-6.11)$

${ }^{\mathrm{c}} P=0.002$, OR: $6.19(95 \% \mathrm{Cl} 1.90-20.18)$

${ }^{\mathrm{d}} P=0.04$, OR: 0.09 (95\% Cl 0.004-1.95).

${ }^{\mathrm{e}} \mathrm{P}=0.04$; OR: 2.01 (95\% Cl 1.02-3.98).

${ }^{\mathrm{f}} P=0.0007$; OR: 4.98 (95\% Cl: $\left.1.88-13.23\right)$.

${ }^{\mathrm{g}} \mathrm{P}=0.006$; OR: 0.25 (95\% Cl: $\left.0.09-0.7\right)$. 
The main purpose of this study was to examine differences in APOE polymorphism between PHN sufferers and non-sufferers; however, as all of these subjects had suffered from shingles we combined these two groups to ascertain information on the distribution of APOE alleles in sufferers from shingles. As this study specifically sought PHN sufferers, these subjects are overrepresented in our cohort. Published values estimate the proportion of PHN in shingles sufferers to be around $20 \% .^{12,13}$ We used this percentage to adjust our values so that a more representative shingles group could be obtained. Specifically, the values for PHN allele (or genotype) frequency were multiplied by 0.2 and added to the values for non-PHN allele (or genotype) frequency multiplied by 0.8 . The modified allele and genotype frequencies are shown in Table 2. Comparing these modified frequencies with those of our control population (Table 3 ) reveals that the APOE- $\varepsilon 4 \varepsilon 4$ frequency is significantly higher for shingles sufferers, suggesting that this genotype is a risk for the disorder, and separation by gender shows that the association is present in females but not in males. It should be noted, however, that the proportion of APOE- $\varepsilon 4$ homozygotes in the population is small (approx $2 \%{ }^{14}$ ) and thus $\varepsilon 4$ on its own could not account for the incidence of shingles, that is, other factors must play a role also. In any case, as the number of APOE- $\varepsilon 4 \& 4$ possessors is low here, a study with larger numbers of shingles patients, and with controls more closely age-matched, would be needed to allow a firm conclusion. There are no associations of allele frequency for any group.

We investigated also APOE genotype and allele frequencies in sufferers of IM (Table 4). This disease is caused by EBV infection in adolescence, so the appropriate control group would consist of those infected in adolescence who did not develop IM, but this group would be extremely difficult to obtain. We therefore compared the APOE values of the IM sufferers with our control population (Table 3). No statistically significant differences were found between the two groups as a whole but division by gender shows a higher APOE- $\varepsilon 4 \varepsilon 4$ frequency in females with IM. However, the number of sufferers are too small to draw firm conclusions.

Our data (summarised in Table 5) thus suggest first that the effects of APOE on VZV are due to those in females though modulated in some cases by a null effect in males; second, for females, APOE- $\varepsilon 4 \varepsilon 4$ may be a risk for shingles and also for IM; third, for females, $\varepsilon 3$ is a risk for PHN whereas $\varepsilon 4$ is protective. However, we stress that the number of people we studied are mostly low, and so these data need to be extended by examining larger numbers.

Table 2 APOE genotype (a) and allele (b) frequencies (weighted) of shingles sufferers

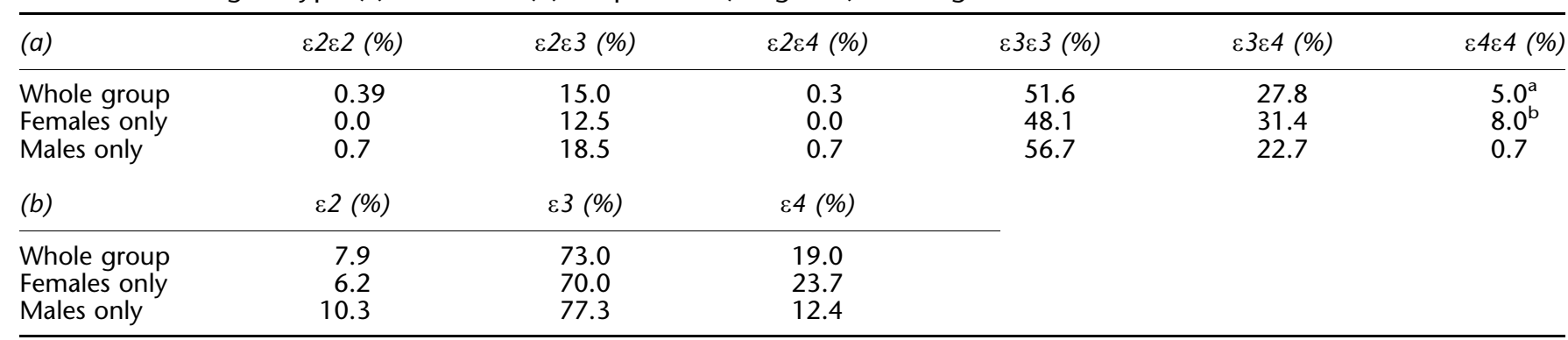

Footnotes display statistics only for those values that differ significantly from the controls, that is $P \leq 0.05$.

${ }^{\mathrm{a}} P=0.008$, OR: $8.17(95 \% \mathrm{Cl} 1.32-50.58)$

${ }^{\mathrm{b}} P=0.003$, OR: $21.6(95 \% \mathrm{Cl} 1.25-373.6)$.

Table 3 APOE genotype (a) and allele (b) frequencies of the control group

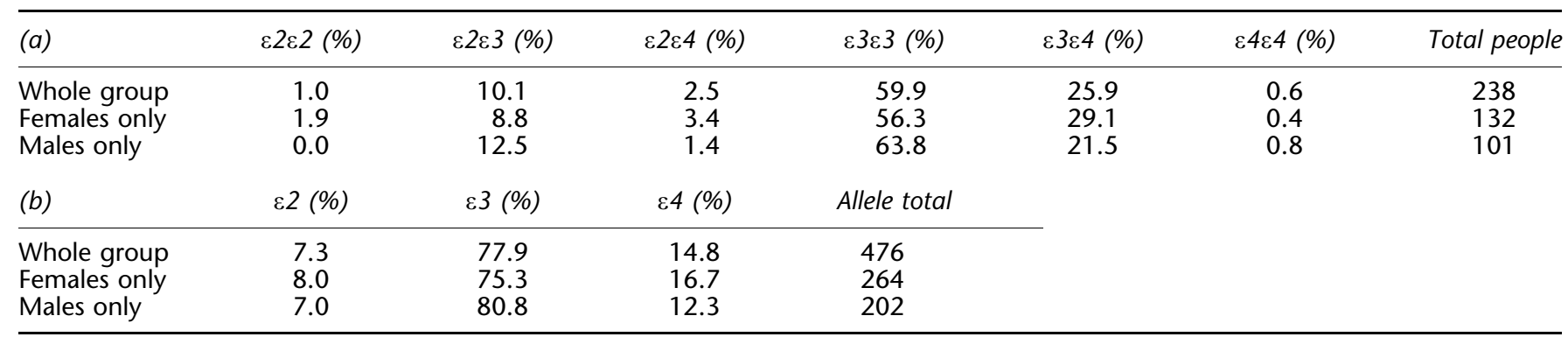


Table 4 APOE genotype (a) and allele (b) frequencies of the infectious mononucleosis sufferers

\begin{tabular}{|c|c|c|c|c|c|c|c|c|c|c|c|c|c|}
\hline \multirow[b]{2}{*}{ (a) } & \multicolumn{2}{|c|}{$\varepsilon 2 \varepsilon 2$} & \multicolumn{2}{|c|}{$\varepsilon 2 \varepsilon 3$} & \multicolumn{2}{|c|}{$\varepsilon 2 \varepsilon 4$} & \multicolumn{2}{|c|}{$\varepsilon 3 \varepsilon 3$} & \multicolumn{2}{|c|}{$\varepsilon 3 \varepsilon 4$} & \multicolumn{2}{|c|}{$\varepsilon 4 \varepsilon 4$} & \multirow[b]{2}{*}{ Total people } \\
\hline & $\mathrm{N}$ & $\%$ & $N$ & $\%$ & $N$ & $\%$ & $\mathrm{~N}$ & $\%$ & $\mathrm{~N}$ & $\%$ & $\mathrm{~N}$ & $\%$ & \\
\hline $\begin{array}{l}\text { Whole group } \\
\text { Females only } \\
\text { Males only }\end{array}$ & $\begin{array}{l}0 \\
0 \\
0\end{array}$ & $\begin{array}{l}0.0 \\
0.0 \\
0.0\end{array}$ & $\begin{array}{l}6 \\
3 \\
3\end{array}$ & $\begin{array}{l}15.4 \\
14.3 \\
16.7\end{array}$ & $\begin{array}{l}1 \\
0 \\
1\end{array}$ & $\begin{array}{l}2.6 \\
0.0 \\
5.6\end{array}$ & $\begin{array}{l}23 \\
11 \\
12\end{array}$ & $\begin{array}{l}59.0 \\
52.4 \\
66.7\end{array}$ & $\begin{array}{l}7 \\
5 \\
2\end{array}$ & $\begin{array}{l}17.9 \\
23.8 \\
11.1\end{array}$ & $\begin{array}{l}2 \\
2 \\
0\end{array}$ & $\begin{array}{l}5.1^{\mathrm{a}} \\
9.5^{\mathrm{b}} \\
0.0^{0}\end{array}$ & $\begin{array}{l}39 \\
21 \\
18\end{array}$ \\
\hline (b) & $\mathrm{N}$ & $2 \%$ & $N$ & $\%$ & $\mathrm{~N}$ & $\%$ & Allele total & & & & & & \\
\hline $\begin{array}{l}\text { Whole group } \\
\text { Females only } \\
\text { Males only }\end{array}$ & $\begin{array}{l}7 \\
3 \\
4\end{array}$ & $\begin{array}{r}9.0 \\
7.1 \\
11.1\end{array}$ & $\begin{array}{l}59 \\
30 \\
29\end{array}$ & $\begin{array}{l}75.6 \\
71.4 \\
80.6\end{array}$ & $\begin{array}{r}12 \\
9 \\
3\end{array}$ & $\begin{array}{r}15.4 \\
21.4 \\
8.3\end{array}$ & $\begin{array}{l}78 \\
42 \\
36\end{array}$ & & & & & & \\
\hline
\end{tabular}

Footnotes display statistics only for those values that differ significantly from the controls, that is $P \leq 0.05$.

${ }^{\mathrm{a}} P=0.02$, OR: $8.4(95 \% \mathrm{Cl} 0.99-71.3)$.

${ }^{\mathrm{b}} P=0.002$, OR: 26.1 (95\% Cl 1.22-560.3).

Table 5 Summary of the findings in this study and in previous APOE polymorphism studies

\begin{tabular}{llll}
\hline Disease & Risk & Protective & Reference \\
\hline Post-herpetic neuralgia & APOE $\varepsilon 3^{\mathrm{a}}$ & APOE $\varepsilon 4^{\mathrm{b}}$ & This study \\
Shingles & APOE $\varepsilon 3 \varepsilon 3^{\mathrm{b}}$ & APOE $\varepsilon 2 \varepsilon 3^{\mathrm{c}}$ & This study \\
Infectious mononucleosis & APOE $\varepsilon 4 \varepsilon 4^{\mathrm{a}}$ & & This study \\
Cold sores & APOE $\varepsilon 4 \varepsilon 4^{\mathrm{b}}$ & & 2,3 \\
Herpes simplex encephalitis & APOE $\varepsilon 4$ & & 4 \\
HCV-induced liver disease & APOE $\varepsilon 2$ & APOE $\varepsilon 4$ & 5 \\
Malaria & APOE $\varepsilon 2 \varepsilon 2$ & & 6 \\
\hline
\end{tabular}

Whole group and females.

bemales only.

'Whole group only.

\section{Discussion}

APOE modulates the outcome of infection with several pathogens. ${ }^{2,4-6}$ The common feature linking these agents is their use of the same molecules in the cell surface for binding and/or entry as those used by apoE. We have hypothesised that different apoE isoforms might differ in respect to binding/entry and thus differentially compete with the infectious agents in these processes, the differences depending on the cell type. This would lead to greater pathogen spread and damage, and hence greater disease, in individuals who possess the less competitive isoforms. Our current study has explored this further by investigating the effects of APOE polymorphism on the consequences of infection with two other viruses, VZV and EBV.

Our main finding is that in female PHN sufferers, the APOE- $\varepsilon 3$ allele is a risk while $\varepsilon 4$ is protective. There are several possible mechanisms to account for this result. First, there could be competition of apoE with VZV. Interestingly, Winkler et $a l^{15}$ have shown with cultures of human skin fibroblasts that apoE isoforms differ in their ability to bind HSPG in the order apoE4 > apoE3 > apoE2.
This type of effect could account for our PHN results in that in APOE- $\varepsilon 4$ carriers, apoE would be more competitive and thus would better prevent entry of VZV. A second possibility relates to the likely involvement of the immune system in PHN; this was proposed because it is uncertain whether or not the virus persists during the disease. ${ }^{16}$ On this basis, the role of apoE in the immune system could provide an alternative explanation. This APOE suppresses proliferation and activation of $\mathrm{T}$ cells and the extent of suppression would depend on the concentration of apoE in plasma. APOE- $\varepsilon 4$ carriers have the lowest concentration, ${ }^{1}$ so their immune cell proliferation and activation would be less than that of carriers of the other alleles. Therefore if PHN is immune cell-mediated, APOE- $\varepsilon 4$ carriers, with a lesser proliferation of T cells, are less likely to develop PHN; however, APOE- $\varepsilon 2$ carriers, with the highest plasma level, would have reduced clearance of VZV and greater occurrence of PHN - and neither is the case. A third possible mechanism involves nitric oxide (NO): NO inhibits the immune system cells that control viral infection, including VZV infection. ${ }^{17}$ As the different apoE isoforms differentially stimulate release of $\mathrm{NO}$ in the order 
apoE3 $>$ apoE $2 \gg$ apoE $4,{ }^{18}$ this could explain why $\varepsilon 4$ is protective and $\varepsilon 3$ is a risk for PHN.

Our results also show that the risk of shingles is conferred by homozygosity for APOE- $\varepsilon 4$. This is seemingly paradoxical in view of the protective effect of the APOE- $\varepsilon 4$ allele in PHN; however, our data show that shingles can also occur in those without an APOE- $\varepsilon 4$ allele and presumably it is these people who would be susceptible to PHN whereas those with an $\varepsilon 4$ allele would be protected. Mechanistically, the risk of shingles conferred by homozygosity for APOE- $\varepsilon 4$ might be explained by a competition effect. Competition effects could account for the APOE- $\varepsilon 4$ links in shingles as well as PHN since previous studies have shown that binding of the apoE isoforms is cell-type dependent, ${ }^{19,20}$ and the types differ between PHN and shingles, the former involving nerve cells whereas the latter involves skin.

The APOE effect we have found in PHN is genderdependent but the reason is unknown. We revealed another example of this dependence when we re-analysed our data on liver damage caused by hepatitis $C$ virus: $:^{5}$ the protective effect of APOE- $\varepsilon 4$ we had found is much greater for males than for females. No other studies have been published on gender effects of APOE in infectious diseases, but they have been shown in certain other types of disease such as some cancers, ${ }^{21}$ and have generally (and vaguely) been attributed to sex hormones. This might be the case in infectious diseases too. Alternatively, APOE's role in the immune system (in which there are known gender effects) might be involved. Another possibility relevant to the entry of apoE (in the form of lipoproteins) into cells is that lipoprotein levels in serum might be gender-dependent. ${ }^{22}$

Very few studies apart from ours (see Table 5) have been made on APOE polymorphisms and infectious diseases, irrespective of gender. The sole previous investigation of shingles and $\mathrm{PHN}^{23}$ detected no differences in APOE allele frequencies from those of controls, ${ }^{24}$ but full data on alleles, genotypes and gender were not given, so only a partial comparison can be made with our results. Their $\varepsilon 4$ frequencies were similar for PHN and non-PHN (14 and $15 \%$ respectively), whereas their $\varepsilon 2$ frequencies were higher for PHN than for non-PHN (9 and 3\% respectively), but the difference was not statistically significant; also, their overall shingles values did not differ significantly from those of their controls. The differences between the study by Pirttilä et $a l^{23}$ and ours might reflect the known differences between APOE frequencies of Finns and other Northern European population, the Finns having higher $\varepsilon 4$ and lower $\varepsilon 2$ allele frequencies. Also Pirtillä et $a l^{23}$ used a phenotyping method that is particularly prone to errors. ${ }^{25}$

As to involvement of APOE in occurrence of chickenpox, the very high prevalence - over $80 \%$ - of VZV infection in children (due to its transmissibility via the respiratory route, unlike other herpesviruses), makes any involvement unlikely, and this was indeed shown to be the case in a preliminary study of a cohort of several hundred children
(The Avon Longitudinal Study of Parents and Children (ALSPAC)) (R Wynn-Jones and RF Itzhaki, unpublished data). The study also suggests that the first episode of cold sores, caused by the equally common herpesvirus, HSV1, occurs at an older age than does chicken pox, but in APOE$\varepsilon 4$ carriers it occurs at a younger age than in those with the other alleles (and also that APOE- $\varepsilon 2$ may be protective). This difference between the effects of HSV1 and VZV is explainable, as the former is less infectious, and the age of primary infection with this virus is rising with rising socioeconomic level.

In conclusion, our results showing that a host genetic factor influences the development of PHN (and suggesting that this is an effect solely in females), is significant not only at the fundamental level of virus-host interaction, but also clinically: if the results were substantiated in a larger study, they could lead to identification of those (female) patients at greater risk of PHN, thus enabling these people - the numbers of whom are likely to increase with increasing longevity - to be targeted for treatment with the most effective drugs.

\section{Acknowledgements}

We thank Mrs Ann Cookson for her excellent technical assistance, the Occupational Health Section for taking blood for the IM studies, Dr Brian Faragher (Liverpool School of Tropical Medicine) for advice on statistical analysis, and Sir Halley Stewart Trust for financial support.

\section{References}

1 Mahley RW, Rall Jr SC: Apolipoprotein E: far more than a lipid transport protein. Annu Rev Genomics Hum Genet 2000; 1: 507-537.

2 Itzhaki RF, Lin WR, Shang D, Wilcock GK, Faragher B, Jamieson GA: Herpes simplex virus type 1 in brain and risk of Alzheimer's disease. Lancet 1997; 349: 241-244.

3 Lin WR, Graham J, MacGowan SM, Wilcock GK, Itzhaki RF: Alzheimer's disease, herpes virus in brain, apolipoprotein E4 and herpes labialis. Alzheimer's Reports 1998; 1: 173-178.

4 Lin WR, Wozniak MA, Esiri MM, Klenerman P, Itzhaki RF: Herpes simplex encephalitis: involvement of apolipoprotein E genotype. I Neurol Neurosurg Psychiatry 2001; 70: 117-119.

5 Wozniak MA, Itzhaki RF, Faragher EB, James MW, Ryder SD, Irving WL: Apolipoprotein E-epsilon 4 protects against severe liver disease caused by hepatitis $C$ virus. Hepatology 2002; 36: 456-463.

6 Wozniak MA, Faragher EB, Todd JA, Koram KA, Riley EM, Itzhaki RF: Does apolipoprotein E polymorphism influence susceptibility to malaria? J Med Genet 2003; 40: 348-351.

7 Dobson CB, Sales SD, Hoggard P, Wozniak MA, Crutcher KA: The receptor-binding region of human apolipoprotein $\mathrm{E}$ has direct anti-infective activity. I Infect Dis 2006; 193: 442-450.

8 Zhu Z, Gershon MD, Ambron R, Gabel C, Gershon AA: Infection of cells by varicella zoster virus: inhibition of viral entry by mannose 6-phosphate and heparin. Proc Natl Acad Sci USA 1995; 92: $3546-3550$.

$9 \mathrm{Li} \mathrm{Q}$, Ali MA, Cohen JI: Insulin degrading enzyme is a cellular receptor mediating varicella-zoster virus infection and cell-to-cell spread. Cell 2006; 127: 305-316.

10 Lin WR, Tullo AB, Itzhaki RF: Apolipoprotein $\mathrm{E}$ and herpes virus diseases: herpes simplex keratitis. Eur J Hum Genet 1999; 7: 401-403.

11 Wenham PR, Price WH, Blandell G: Apolipoprotein E genotyping by one-stage PCR. Lancet 1991; 337: 1158-1159. 
12 Coen PG, Scott F, Leedham-Green M et al: Predicting and preventing post-herpetic neuralgia: Are current risk factors useful in clinical practice? Eur J Pain 2006; 10: 695-700.

13 Scott FT, Johnson RW, Leedham-Green M, Davies E, Edmunds WJ, Breuer J: The burden of Herpes Zoster: a prospective population based study. Vaccine 2006; 24: 1308-1314.

14 Weintraub MS, Eisenberg S, Breslow JL: Dietary fat clearance in normal subjects is regulated by genetic variation in apolipoprotein E. J Clin Invest 1987; 80: 1571-1577.

15 Winkler K, Scharnagl H, Tisljar U et al: Competition of Abeta amyloid peptide and apolipoprotein $\mathrm{E}$ for receptor-mediated endocytosis. J Lipid Res 1999; 40: 447-445.

16 Gilden DH, Cohrs RJ, Mahalingam R: VZV vasculopathy and postherpetic neuralgia: progress and perspective on antiviral therapy. Neurology 2005; 64: 21-25.

17 Ito M, Watanabe M, Kamiya H, Sakurai M: Inhibition of natural killer (NK) cell activity against varicella-zoster virus (VZV)infected fibroblasts and lymphocyte activation in response to VZV antigen by nitric oxide-releasing agents. Clin Exp Immunol 1996; 106: 40-44.

18 Sacre SM, Stannard AK, Owen JS: Apolipoprotein E (apoE) isoforms differentially induce nitric oxide production in endothelial cells. FEBS Lett 2003; 540: 181-187.

19 Mamotte CD, Sturm M, Foo JI, van Bockxmeer FM, Taylor RR: Comparison of the LDL-receptor binding of VLDL and LDL from
apoE4 and apoE3 homozygotes. Am J Physiol 1999; 276: E553E557.

20 Bohnet K, Pillot T, Visvikis S, Sabolovic N, Siest G: Apolipoprotein (apo) E genotype and apoE concentration determine binding of normal very low density lipoproteins to HepG2 cell surface receptors. J Lipid Res 1996; 37: 1316-1324.

21 Watson MA, Gay L, Stebbings WS, Speakman CT, Bingham SA, Loktionov A: Apolipoprotein E gene polymorphism and colorectal cancer: gender-specific modulation of risk and prognosis. Clin Sci (Lond) 2003; 104: 537-545.

22 Freedman DS, Otvos JD, Jeyarajah EJ et al: Sex and age differences in lipoprotein subclasses measured by nuclear magnetic resonance spectroscopy: the Framingham Study. Clin Chem 2004; 50: $1189-1200$

23 Pirttila T, Haanpaa M, Mehta PD, Lehtimaki T: Apolipoprotein E (APOE) phenotype and APOE concentrations in multiple sclerosis and acute herpes zoster. Acta Neurol Scand 2000; 102: 94-98.

24 Nikkila M, Pitkajarvi T, Koivula T et al: Women have a larger and less atherogenic low density lipoprotein particle size than men. Atherosclerosis 1996; 119: 181-190.

25 Snowden C, Houlston RS, Arif MH, Laker MF, Humphries SE, Alberti KG: Disparity between apolipoprotein E phenotypes and genotypes (as determined by polymerase chain reaction and oligonucleotide probes) in patients with non-insulin-dependent diabetes mellitus. Clin Chim Acta 1991; 196: 49-57. 